\title{
CAMPANHA NACIONAL DE ALERTA AO ZUMBIDO: RELATO DE EXPERIÊNCIA NA ATENÇÃO PRIMÁRIA À SAÚDE
}

\section{NATIONAL ZUMBID ALERT CAMPAIGN: EXPERIENCE REPORT IN PRIMARY HEALTH CARE}

Paôlla Gabrielly Antas Lunguinho Dantas ${ }^{1}$ Maria Júlia Galindo Soares ${ }^{2}$ Mariana Nunes Azevedo de Melo $^{3}$ Janaína von Söhsten Trigueiro ${ }^{4}$

RESUMO: OBJETIVO: Com o propósito de fortalecer a Campanha Nacional de Alerta ao Zumbido, objetivou-se sensibilizar a comunidade acerca da ocorrência do zumbido, com a finalidade de viabilizar o diagnóstico precoce e demonstrar a importância do fonoaudiólogo no cuidado a esse sintoma. MÉTODO: Este estudo é um relato de experiência de uma ação educativa apoiadora da Campanha Nacional de Alerta ao Zumbido realizada na sala de espera de uma Unidade de Saúde da Família (USF) do município de João Pessoa-PB durante o Estágio Supervisionado em Saúde Coletiva. RESULTADOS: A ação desenvolvida possibilitou um contato direto com a comunidade, além de funcionários e demais estagiários. Houve grande atenção por parte de todos acerca do tema, por ser sobre um sintoma bastante frequente na rotina dos ouvintes e pouco abordado no cotidiano. A sala de espera subsidia ações baseadas nos pilares do SUS e da Política de Saúde vigente e se mostra como um espaço potente para a educação em saúde, com isso, os profissionais da área devem explorar mais esse locus para promoção e favorecimento do letramento em saúde. CONCLUSÃO: Foi criada uma atmosfera propícia para a horizontalização do aprendizado vivo, em constante movimento. A manutenção de ações na sala de espera se faz pertinente, por ser um método simples, resolutivo e acessível, trazendo mais conforto e proximidade dos

\footnotetext{
${ }^{1}$ Graduanda do curso de Fonoaudiologia da Universidade Federal da Paraíba - UFPB, João Pessoa Paraíba, Brasil. E-mail: paollagabrielly19@gmail.com.

${ }^{2}$ Graduanda do curso de Fonoaudiologia da Universidade Federal da Paraíba - UFPB, João Pessoa Paraíba, Brasil. E-mail: juliagalindo8@gmail.com.

${ }^{3}$ Graduanda do curso de Fonoaudiologia da Universidade Federal da Paraíba - UFPB, João Pessoa Paraíba, Brasil. E-mail: mariananadm@gmail.com.

4 Doutora em Enfermagem e Professora Adjunta do Departamento de Fonoaudiologia da Universidade Federal da Paraíba - UFPB, João Pessoa - Paraíba, Brasil. E-mail: janavs_23@hotmail.com.
} 
profissionais com os pacientes. Acresce-se ainda, ser um espaço que pode se moldar a cada atividade, abrangendo uma gama de temáticas a serem disseminadas, no caso aqui o zumbido.

Palavras chave: Atenção Primária à Saúde. Educação em Saúde. Fonoaudiologia. Zumbido.

ABSTRACT: OBJECTIVE: With the purpose of strengthening the National Tinnitus Alert Campaign, the objective was to sensitize the community about the occurrence of tinnitus, with the purpose of making the early diagnosis feasible and demonstrating the importance of the speech therapist in caring for this symptom. METHOD: This study is an experience report of an educational action supporting the National Tinnitus Alert Campaign held in the waiting room of a Family Health Unit (USF) in the city of João Pessoa-PB during the Supervised Internship in Public Health. RESULTS: The action developed enabled direct contact with the community, in addition to employees and other interns. There was great attention on the part of everyone on the topic, as it is a symptom that is very common in the listeners' routine and rarely addressed in everyday life. The waiting room subsidizes actions based on the pillars of the SUS and the current Health Policy and shows itself as a potent space for health education, with this, professionals in the area should further explore this locus to promote and favor health literacy. CONCLUSION: A favorable atmosphere was created for the horizontalization of living learning, in constant movement. The maintenance of actions in the waiting room is pertinent, as it is a simple, resolutive and accessible method, bringing more comfort and proximity between professionals and patients. In addition, it is a space that can be adapted to each activity, covering a range of themes to be disseminated, in this case, tinnitus.

Keywords: Primary Health Care. Health Education. Language and Hearing Sciences. Tinnitus. 


\section{INTRODUÇÃO}

Os estágios curriculares supervisionados são frequentemente encontrados nos cursos de licenciatura e nos cursos da área da saúde. Vistos como uma forma de interligar a teoria com a prática e, segurados por profissionais experientes e capacitados, os discentes têm a oportunidade de compreender melhor a realidade da profissão e aperfeiçoam o desempenho nas áreas de atuação (SCALABRIN; MOLINARI, 2013).

A Saúde Coletiva, em sua práxis, se constitui em dois planos, o vertical e o horizontal. O primeiro se baseia na intervenção, enquanto o segundo se caracteriza pelas práticas e saberes de todos os agentes envolvidos. Assim, é preciso que as profissões da saúde abranjam na formação e na prática os elementos que compõem tal âmbito de atuação (OSMO; SCHRAIBER, 2015).

Nesse contexto, Becker e Rocha (2017) apontam a importância da criação e fortalecimento de grupos de sala de espera. Essa iniciativa viabiliza a autonomia dos usuários, da cidadania, promove trocas de saberes e ainda fortalece o vínculo entre usuários e equipe de saúde. Ademais, tenta se articular com a necessidade da população alvo e reconhecer a cultura vigente, de modo que proporcione a interação dialógica.

Quanto ao crescimento da Fonoaudiologia na saúde coletiva, um dos marcos foi a criação de uma rede de centros de referência direcionados à saúde do trabalhador. Reconheceu- se que tal profissão poderia contribuir com assistência a essa população específica, participação em estudos e pesquisas, de ações de vigilância, avaliando impactos das tecnologias na saúde, dentre outros (BERNARDI, 2007).

A entrada do fonoaudiólogo na Atenção Primária à Saúde (APS) foi potencializada com a publicação da Portaria n 154 em 24 de janeiro de 2008, a qual criou os Núcleos de Apoio à Saúde da Família (NASF) com o objetivo de solidificar 
esse nível de atenção no Brasil por meio do aumento das ofertas de saúde na rede de serviço (BRASIL, 2008).

Os fonoaudiólogos no NASF vêm desenvolvendo diversas atividades, tais como diagnóstico de saúde do território em que estão inseridos, visitas domiciliares, realizando atendimentos individuais e coletivos, etc. Ainda participam de reuniões multidisciplinares sobre discussão de casos e, desse modo, conseguem atender parte da demanda preconizada na portaria supracitada (FERNANDES; NASCIMENTO; SOUSA, 2013).

Ressalta-se que as competências do profissional fonoaudiólogo na APS, devem ser fundamentadas nas doutrinas e princípios operacionais do Sistema Único de Saúde (SUS) e acatar as propostas das últimas Conferências Mundiais de Saúde (LIPAY; ALMEIDA, 2007). Sendo um dos profissionais que compõem a equipe multidisciplinar que atende pacientes que apresentam zumbido, 0 fonoaudiólogo vem ganhando espaço e reconhecimento nesse campo devido a sua importante contribuição para o controle do sintoma.

O zumbido é um sintoma no qual o paciente relata ouvir um som, porém não há nenhum estímulo externo que esteja produzindo o mesmo. Por se tratar de um sintoma causado por múltiplas etiologias e ter influências pessoais, a intervenção é um desafio para a equipe multidisciplinar que participa desse processo (BRANCOBARREIRO; SANTOS; COELHO, 2015).

Sua ocorrência acontece principalmente em indivíduos a partir de 50 anos e a literatura demonstra que $80 \%$ dos casos são de origem neurossensorial. Destaca-se ainda que a perda auditiva não funciona como um provedor desse sintoma, já que alguns pacientes tem a audição normal e apresentam grau de incomodo significativo, que pode ser associado à adversidades como estresse, ansiedade e depressão (ESTEVES et al., 2012).

A Campanha Nacional de Alerta ao Zumbido foi criada em 2006 pela professora doutora Tanit Ganz Sanchez com o escopo de conscientizar a população sobre o aumento das alterações auditivas em faixas etárias distintas causadas, principalmente, pela poluição sonora e altos níveis de ruído encontrados nas metrópoles, além de incentivar os profissionais de saúde a aderirem a esta causa (INSTITUTO GANZ SANCHEZ, 2019). 
Com o propósito de fortalecer a campanha supracitada no estado da Paraíba aproveitando o espaço ofertado pelo Estágio Supervisionado em Saúde Coletiva, aproveitando o ensejo da Campanha Nacional, objetivou-se sensibilizar a comunidade acerca da ocorrência do zumbido, para com isso, viabilizar o diagnóstico precoce e demonstrar a importância do fonoaudiólogo no cuidado a esse sintoma.

\section{MÉTODO}

Este estudo é um relato de experiência de uma ação educativa apoiadora da Campanha Nacional de Alerta ao Zumbido. Por ocorrer anualmente no mês de novembro, ficou conhecida como Novembro Laranja. A atividade foi realizada na sala de espera de uma Unidade de Saúde da Família (USF) do município de João Pessoa-PB, por alunas do sexto período do curso de graduação em Fonoaudiologia da Universidade Federal da Paraíba (UFPB) durante o Estágio Supervisionado em Saúde Coletiva.

Faz-se mister expor que a atividade proposta fundamentou-se na filosofia freireana, a qual assegura que a educação é horizontal uma vez que tem a capacidade de tornar iguais todos os envolvidos no processo de construção de conhecimento (FREIRE, 1996).

Durante a supervisão do Estágio supracitado, desde o início houve discussões a respeito das possíveis ações a serem desenvolvidas pelas alunas na USF. No mês de novembro foi sugerido, por um membro do grupo, desenvolver uma ação educativa sobre a temática de zumbido, com o intuito de abordar seu conceito, principais características e possíveis causas. Optou-se também por esclarecer sobre o diagnóstico, quando procurar o tratamento e quais profissionais podem atuar, enfatizando o trabalho do fonoaudiólogo.

Cabe destacar que todas as ações sugeridas passam pela aprovação da equipe e recebe um planejamento, haja vista a dinâmica diária da Unidade que não 
deve ser comprometida com a presença do Estágio, mas sim otimizada, de modo a proporcionar um melhor atendimento para os usuários.

Para a ação em questão foi necessário um planejamento prévio. Foi feita uma pesquisa para aprofundamento sobre o assunto, para melhor domínio das discentes. Houve ainda uma discussão de quais materiais seriam utilizados e, elegeu-se um folder (figura 1) disponível online e gratuitamente pelo Instituto Ganz Sanchez para ser entregue aos sujeitos no momento da atividade educativa. Ademais, foram confeccionadas fitinhas laranja representando a cor do mês de conscientização ao zumbido. 


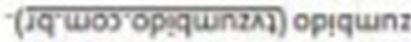
op eano ap soses axqos exyquạs eșnbsad eq̣auyd ep sojuawiodap so ayuedwose 'sasinb as ¡esueyuos

ap oujs.zo oगpą nas wos as-awjoju। วya sompipne soulajede 'sejouor saghejnugsa 'saofesipow 'sajezusuiןe sefuepnu wos oys;

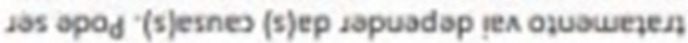
jouाaw op ey|cosa $\forall$ 'anuawjepjed souaw ojad "opiquinz o jesouplaw wapod sespos sejpa 'wis ¿opiqunzo esed oyuawezen asspr3

ossaons ap aouey? e jojew 'ojuawezen o sesawos sazue oquenb soyujzos ejoyןau opiqunz o anb sesuad ayk opiano

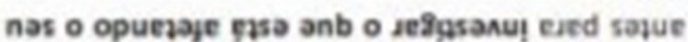

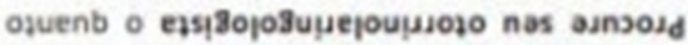
¿oprqunz was opuenb sazejanb 0 "assansa a op̧expaw-ozne

'(еujajes a saoop ap osnqe 'unf(a) sajquawine

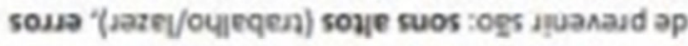
s!̣วey șew sV 'op!qunz ap sesnes sejuen wasspix

coplquanzo jesnes apod anb 0 'opssajdap no apeparsue 'iepos ерн

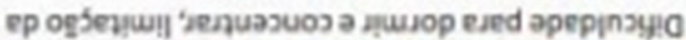
¿opiqunz was wanb asuss anb 0 '(sonquadas a soxieq suos wos opoworul) eluojosןw e'(suos sop aunjon o wos opowpoui) eisnoeradị e "(suos so japuanu no juno ejed apepinayip) expipne epsad e owos 'sopeisosse orjsa _opyno op eylupenb, ep sewosurs sosano sazan seanu toyuzos asajede azuawejey jossip jeje;

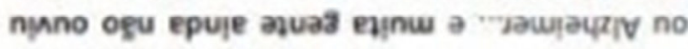

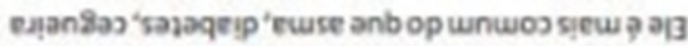

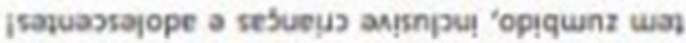
ef apep! danbjenb ap oesejndod ep жoz ap șew ¿opiqum sas wnwos 3

\begin{abstract}
10 DICAS para culdar dos ouvidos!
1. In festas, shows e bares: use proteter de ouvido e faça intervalos peribsicos (10min por hora). Nlo b" normar" sai comrumbido!

2. Música com fones de ouvido evite passar da metade da petincia do apareho 2 h de uso continuo:

1. Nimentese 4 a 6 vetes ao da sem atusar de doces e cafeina.

4 Midrate-se bem para seus rins eliminarem as tosinas.

5. Eserdite-se 5 veas por semana: seu metabolimo va melhorar

6. Alvie sev estresse com abvidades relacuntes.

7. Istimule seus ouvidos com baixo volume de sons suaves.

B. Redura ocontato do celular com o owido.

9 . Eite auto medicagle

10. Crie momentos de felicidade: eles ajudam a restaurat os órslos, inclusive os ouvidos.

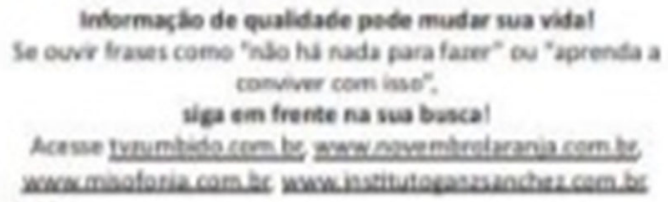

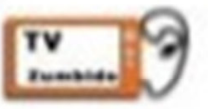

Ganz sincilz

\section{Mais de $\mathbf{4 0}$ milhões de brasileiros de todas as idades têm zumbido. Você ou seus filhos estão entre eles?}

O Nevembro Laranja e uma campanha de
Neventre Lankwis CAMPANHA NACIONAL OE ALERTA AO ZUMBIDO

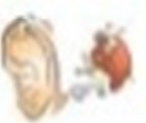
divulgaclo da "quadrilha de ouvids". Saba que: 1. Zumbido, misotonia, hiperacusia, perda auditiva e tentura so sintemas de ouvidos vulererveis,

2. As causas grecisam de imesticaclo correta para serem descobertas: 3. O tratamento precoce pode tarer a diferenca na recuperagko do ouvido.

Oque to rumbide?

f o som que as pessoas escutam derntro dos ouvidos. especialmente no slitncio (apino, chiado, cigarra etc). rie e um "sinal de alerta" de agresslo ao ouvido.

Figura 1 - Folder da Campanha - Nacional de alerta ao zumbido. 
A ação contou com a participação de usuários da USF, funcionários e alunos dos cursos de Enfermagem e Nutrição que também realizam estágio na Unidade. $\mathrm{Na}$ ocasião foi abordado o tema proposto, o qual teve grande impacto e desenvolveu entusiasmo dos participantes, sobretudo por terem ou conhecerem algum familiar ou pessoa próxima que apresenta o sintoma do zumbido.

Em momento oportuno foi divulgado o trabalho realizado pelo Grupo de Estudo e Pesquisa em Audiologia, Equilíbrio e Zumbido (GEPAEZ), coordenado por uma fonoaudióloga docente da UFPB. Nele é oferecido diagnóstico e tratamento gratuito na Clínica Escola de Audiologia, sendo disponibilizada aos pacientes uma equipe multiprofissional.

O GEPAEZ organiza encontros mensais com os pacientes de zumbido e ainda desenvolve anualmente o Encontro Multidisciplinar sobre Zumbido. Algo que precisa ser enaltecido foi a parceria feita com a Equipe Literacia de Inovação e Tecnologia em Saúde (ELITS), a qual rendeu um aplicativo que facilita o diagnóstico do zumbido, reforçando o comprometimento e cuidado de toda equipe para a acessibilidade e qualidade dos serviços ofertados. 


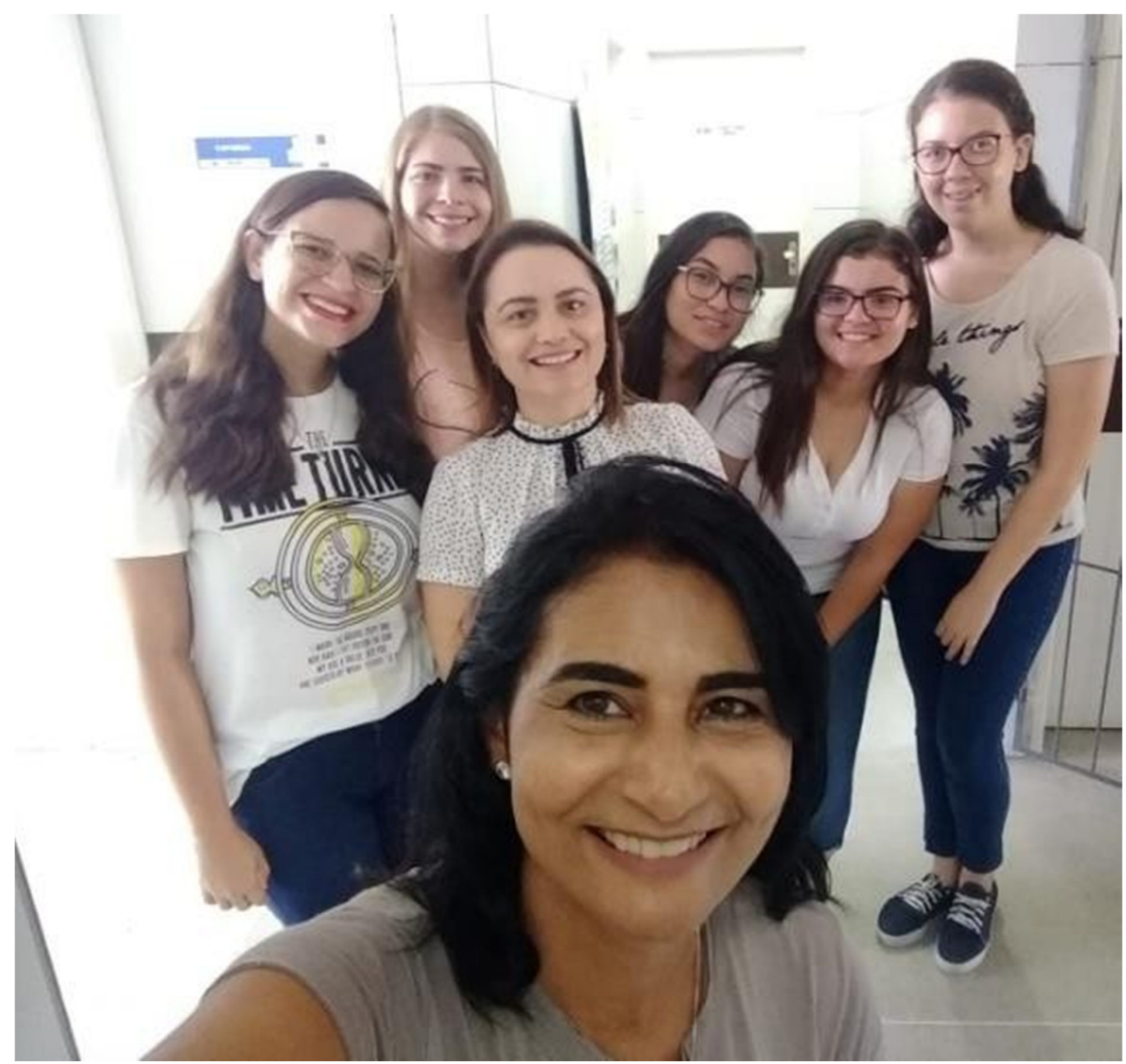

Figura 2 - Grupo de estagiárias, professora supervisora e a preceptora do serviço.

\section{RESULTADOS E DISCUSSÃO}

A ação desenvolvida (figura 3) possibilitou um contato direto com a comunidade, além de funcionários e demais estagiários. Houve grande atenção por parte de todos acerca do tema, por ser sobre um sintoma bastante frequente na 
rotina dos ouvintes e pouco abordado no cotidiano. Com isso, auxilia na educação em saúde e na construção de espaços que permitem esta prática, promove uma formação dos profissionais de saúde diversificada, além de investir na autonomia da sociedade para que esta assuma decisões sobre sua vida e saúde (ROSA; BARTH; GERMANI, 2011).

Unir a visão preventiva e a promoção da saúde pela atividade educativa à rotina curativa é primordial para a efetividade do serviço. A partir do conhecimento disponível e acessível, o sujeito pode ser conscientizado e mudar hábitos e comportamentos (ZAMBENEDETTI, 2012). A sala de espera subsidia ações baseadas nos pilares do SUS e da Política de Saúde vigente e se mostra como um espaço potente para a educação em saúde, com isso, os profissionais da área devem explorar mais esse locus para promoção e favorecimento do letramento em saúde (REIS et al., 2014).

A sala de espera é um ambiente propício por estreitar laços com o usuário e proporcionar um momento de acolhimento para a população. No geral, é um serviço que agrada a população (84,6\% consideram excelente ou boa), contradizendo as opiniões existentes sobre estrutura ( $70 \%$ fariam mudanças), já que são realizadas em ambientes com uma poluição sonora considerável e pouco conforto, como mostram Silva et al.(2019) em seu estudo.

Foi elucidado acerca das especificidades do sintoma para que pudesse ser facilmente identificado. Alertou-se sobre as principais causas e como evitar possíveis danos à audição, já que a maioria dos pacientes de zumbido apresenta alteração neurossensorial. É válido ressaltar que para um diagnóstico confiável, é indispensável a realização de diversos testes.

Um dado importante foi que vários usuários presentes na ação conheciam ou se reconheceram como pessoas que apresentam zumbido. Em virtude disso, houve a orientação sobre o trabalho desenvolvido pelo GEPAEZ a fim de que sejam tomados os procedimentos cabíveis para o diagnóstico adequado e atendimento ofertado pela equipe, lembrando que há a necessidade de articulação entre a rede de saúde pública e a Universidade. 


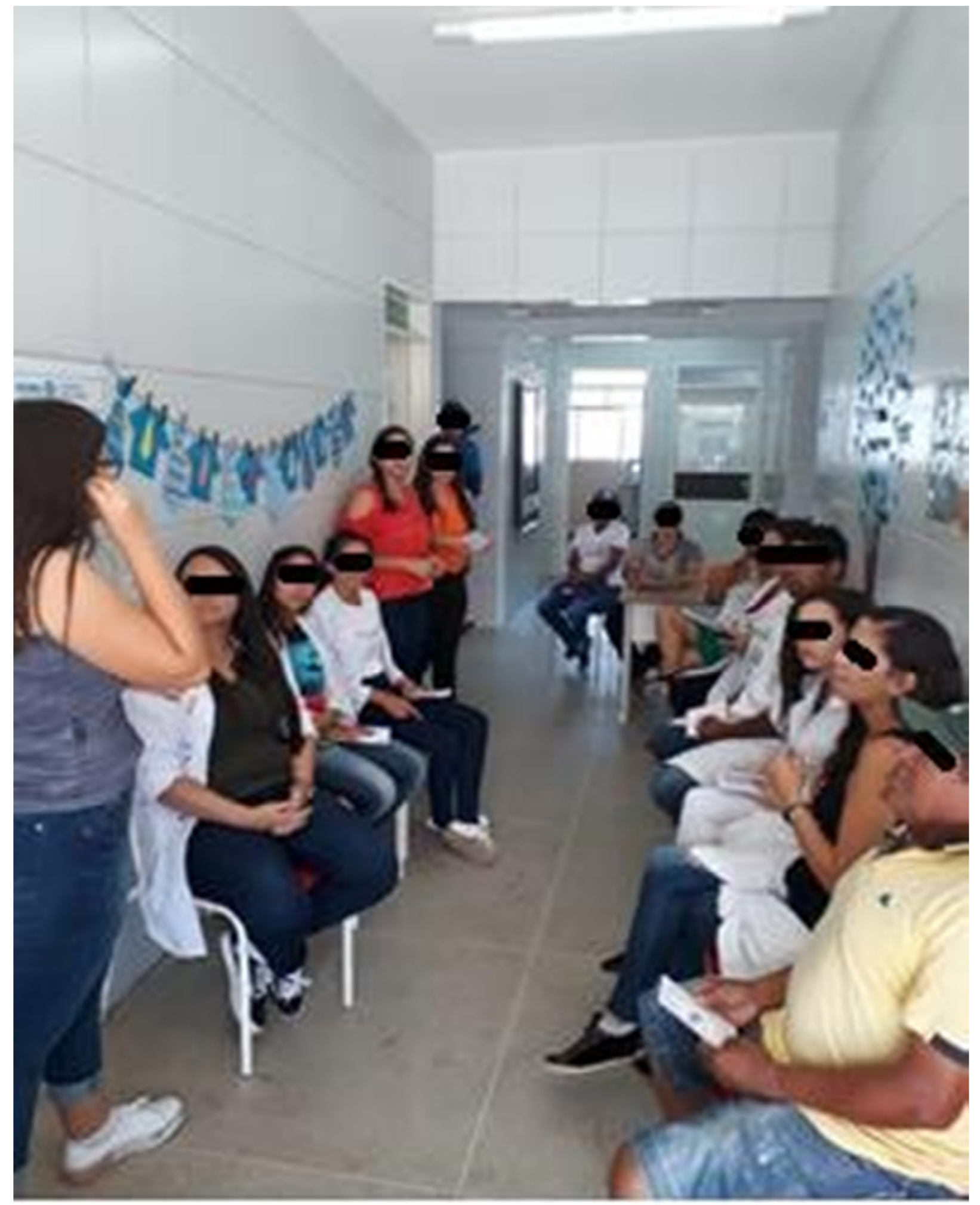

Figura 3 - Um dos momentos da educação em saúde na sala de espera. 
As ações realizadas na APS devem fortalecer autonomia do coletivo, entretanto, a participação comunitária está sendo pouco expressiva, conforme Prado e Santos (2018). Em sua maioria, estão com foco voltado às doenças crônicas e mudanças de hábitos de vida inadequados, deixando de lado a promoção da saúde da população saudável (KESSLER et al., 2018). Embora permita a interação do alunado com a comunidade, proporcione trabalho de educação em saúde, favoreça a divulgação da atuação profissional e encaminhe casos que necessitem de atendimento especializado há dificuldades, tais como: ambiente ruidoso e o trânsito de usuários que não desejam participar das ações (TEIXEIRA; VELOSO, 2006).

Uma informação que necessita ser evidenciada é a criação da Política Nacional de Atenção à Saúde Auditiva, em 2004, pelo Ministério da Saúde (MS). Tal avanço permitiu a implementação de ações direcionadas à saúde auditiva no âmbito do SUS (ROSSI BARBOSA et al., 2010). Todavia, Lacerda et al. (2013) apontam que na Fonoaudiologia, a problematização ainda é incipiente no processo educativo por meio da promoção da saúde, o que torna a ação realizada pelo Estágio Supervisionado em Saúde Coletiva ainda mais relevante.

Outro ponto que deve ser mencionado foi a efetiva participação dos Agentes Comunitários de Saúde (ACS) durante a ação educativa. Todos estavam bastantes interessados em aprender mais sobre o tema em questão bem como preocupados com alguns idosos de suas áreas de abrangência que apresentavam zumbido. Nessa perspectiva, a pesquisa de Santos et al. (2012) demonstrou que a maioria dos ACS reconhecem algumas situações de risco à saúde fonoaudiológica, principalmente àquelas relacionadas às áreas da voz e motricidade orofacial.

Contudo, enfatiza-se ser fundamental que esses profissionais sejam capacitados continuadamente para que sejam aptos a detectar, informar e sensibilizar tanto a comunidade quanto a equipe, tornando-se agentes potenciais de promoção da saúde e fortes aliados à Fonoaudiologia.

Corroborando tal afirmativa, Ribeiro et al. (2014) relatam que capacitações em saúde auditiva são imprescindíveis em todos os âmbitos de atuação do fonoaudiólogo, nomeadamente nas Unidades de Saúde, dada a sua relevância no processo comunicativo e social dos indivíduos. Porém, as atividades direcionadas para a promoção e prevenção de alterações nessa área são limitadas e, mais uma 
vez, constata-se o quão importante foi realizar ações desse tipo na APS, especialmente por discentes que ainda estão em formação e podem, na sua futura atuação profissional, fazerem a diferença.

Fazendo alusão à formação acadêmica do fonoaudiólogo, observa-se que a partir dos marcos conceituais da promoção de saúde, enaltece-se então, a relevância da formação dos diversos profissionais da área sendo articulada com as políticas de saúde pública, com a finalidade de desenvolver as competências necessárias para atuar no SUS (CASANOVA; MORAES; RUIZ-MORENO, 2010). Como consequência, se inserem no mercado diferentes núcleos de saberes que se unem com o propósito de cuidar do indivíduo, que é plural na sua singularidade.

É nessa conjuntura que vemos a importância de uma equipe multidisciplinar na APS, por ser porta de entrada e está em contato direto com múltiplas demandas. Esse papel é do NASF desde 2008, o qual, segundo a Portaria No 204/GM de 29 de janeiro de 2007, deveria receber recursos federais para as ações e os serviços. Porém, segundo a nota técnica divulgada pelo Ministério da Saúde de № 3/2020DESF/SAPS/MS, agora os gestores municipais e estaduais têm autonomia na aplicação dos incentivos de custeio federal referente ao financiamento.

Isso vem ocasionando preocupação por parte dos que atuam no NASF, pois muitos gestores municipais e estaduais afirmam que o financiamento é insuficiente, e como o financiamento de custeio da APS pode ou não ser aplicado em equipes multiprofissionais, poderá ocasionar demissões em massa, prejudicando, sobretudo o usuário, já que o mesmo será destinado para um serviço especializado, consequentemente, haverá uma alta demanda e encharcar os serviços de atenção secundária e, quiçá, do nível terciário.

Apesar da atenção secundária em saúde desenvolver um papel essencial na resolubilidade e integralidade da Rede de Atenção em Saúde (RAS), ainda necessita aprimorar as ferramentas de planejamento, desde a seleção de protocolos até a ampliar da capacidade dos serviços (ERDMANN et al., 2013).

É notório, portanto, que ações de cunho educativo e de promoção da saúde, como a que foi realizada, sejam cada vez mais estimuladas no sentido de sensibilizar a população e consolidar as atividades que são peculiares à APS, fortalecendo-a como a porta de entrada para a RAS. 
Assim, a metodologia dialógica, respeitando o senso comum, a diversidade cultural e as demandas dos usuários, associada ao conhecimento científico necessário trouxe enriquecimento pessoal e profissional para os participantes da ação, além de promover preparo e instigar a população como seres multiplicadores de informação, tendo em vista a constância do sintoma do zumbido na sociedade.

\section{CONCLUSÃO}

No palco de atuação da APS o grupo de Estágio Supervisionado em Saúde Coletiva, após diversos ensaios na Universidade, se apresentou com louvor. Uma atuação onde ninguém conseguiu diferenciar quem era protagonista ou coadjuvante, pois os saberes comuns e os científicos constituíram, no final, o roteiro ideal para a educação em saúde. Paulo Freire certamente aplaudiria de pé tal iniciativa, uma vez que foi criada uma atmosfera propícia para a horizontalização do aprendizado vivo, em constante movimento.

Lançando mão da prosopopeia, o intuito aqui é deixar clara a importância da educação em saúde, tanto para o desenvolvimento da autonomia do usuário quanto para o preparo da equipe, já que está em contato diário com os sujeitos e precisam saber de informações que auxiliem o acesso integral à saúde. A manutenção de ações na sala de espera se faz pertinente, por ser um método simples, resolutivo e acessível, trazendo mais conforto e proximidade dos profissionais com os pacientes. Acresce-se ainda, ser um espaço que pode se moldar a cada atividade, abrangendo uma gama de temáticas a serem disseminadas, no caso aqui o zumbido.

Reflete-se, por fim, a relevância da ação para a formação das discentes que a planejaram e executaram. Muito além dos aspectos do zumbido, pôde-se experienciar a saúde coletiva no seu cerne junto à equipe da USF, inclusive com fortes traços do trabalho interprofissional, que preza por práticas colaborativas. A comunicação, a criatividade, a sensibilidade, o dinamismo de atividades em conjunto, todos foram aguçados a fim de estabelecer o aprendizado na práxis. 


\section{REFERÊNCIAS BIBLIOGRÁFICAS}

BRANCO-BARREIRO, Fátima Cristina Alves; SANTOS, Gisele Munhóes dos; COELHO, Cláudia Barros. Zumbido| Avaliação e Intervenção. In: BOÉCHAT, Edilene Marchini. Tratado de Audiologia. 2. Ed. Rio de Janeiro: Guanabara Koogan, cap. 70. 2015.

BECKER, Ana Paula Sesti; ROCHA, Natália Lorenzetti da. Ações de promoção de saúde em sala de espera: contribuições da Psicologia. Mental, Barbacena, v. 11, n. 21, p. 339-355, dez. 2017.

BERNARDI, Alice Penna de Azevedo. Editorial II. Revista CEFAC, São Paulo, v. 9, n. 2, Jun. 2007.

BRASIL. Ministério da Saúde. Portaria No 154, de 24 de janeiro de 2008. Cria os Núcleos de Apoio à Saúde da Família - NASF. Disponível em http://bvsms.saude.gov.br/bvs/saudelegis/gm/2008/prt0154_24_01_2008.html. Acesso em: 22 fev. 2020.

BRASIL. Ministério da Saúde. Secretaria de Atenção Primária à Saúde. Departamento de saúde da Família. Nota Técnica No 3/2020-DESF/SAPS/MS. Disponível em: https://www.conasems.org.br/wp- content/uploads/2020/01/NT-NASF-AB-e-Previne-Brasil-1.pdf. Acesso em: 22 fev. 2020.

CASANOVA, Isis Alexandrina; MORAES, Ana Alcídia de Araújo; RUIZ-MORENO, Lidia. O ensino da promoção da saúde na graduação de fonoaudiologia na cidade de São Paulo. ProPosições, Campinas, v. 21, n. 3, p. 219-234, Dez. 2010.

ERDMANN, Alacoque Lorenzini et al. A atenção secundária em saúde: melhores práticas na rede de serviços. Revista Latino-Americana de Enfermagem, Ribeirão Preto, v. 21, n. spe, p. 131-139, Fev. 2013.

ESTEVES, Carolina Campos et al. Audição, zumbido e qualidade de vida: um estudo piloto. Revista CEFAC, São Paulo, v. 14, n. 5, p. 836-843, Out. 2012.

FERNANDES, Thaís de Lima; NASCIMENTO, Cynthia Maria Barboza do; SOUSA, Fabiana de Oliveira Silva. Análise das atribuições dos fonoaudiólogos do NASF em municípios da região metropolitana do Recife. Revista CEFAC, São Paulo, v. 15, n. 1, p. 153-159, fev. 2013.

FREIRE, Paulo. Pedagogia da Autonomia: saberes necessários à prática educativa. 33 ed. São Paulo: Paz e terra, 1996.

Instituto Ganz Sanchez. Campanha Nacional de Alerta ao Zumbido. 2019. Disponível em: https://www.institutoganzsanchez.com.br/novembrolaranja/ Acesso em: 22 fev. 2020.

KESSLER, Marciane et al. Ações educativas e de promoção da saúde em equipes do Programa Nacional de Melhoria do Acesso e da Qualidade da Atenção Básica, Rio Grande do Sul, Brasil. Epidemiologia e Serviços de Saúde, Brasília, v. 27, n. 2, 2018.

LACERDA, Adriana Bender Moreira de et al. Oficinas educativas como estratégia de promoção da saúde auditiva do adolescente: estudo exploratório. Audiology -Communication Research, São Paulo, v. 18, n. 2, p. 85-92, Jun 2013.

LIPAY, Maria Somenzari; ALMEIDA, Elizabeth Crepaldi. A fonoaudiologia e sua inserção na saúde pública. Revista de Ciências Médicas. Campinas, v. 16, n. 1, p. 31-41, fev. 2007. 
OSMO, Alan; SCHRAIBER, Lilia Blima. O campo da Saúde Coletiva no Brasil: definições e debates em sua constituição. Saúde e Sociedade, São Paulo, v. 24, supl. 1, p. 205-218, Jun 2015.

PRADO, Nilia Maria de Brito Lima; SANTOS, Adriano Maia dos. Promoção da saúde na Atenção Primária à Saúde: sistematização de desafios e estratégias intersetoriais. Saúde em debate, Rio de Janeiro, v. 42, n. spe1, p. 379-395, Set. 2018.

REIS, Francine Vieira et al. Educação em saúde na sala de espera - relato de experiência. Revista Médica de Minas Gerais, 4(supl.1), fev. 2014.

RIBEIRO, Gisele Marques; FIGUEIREDO, Maria Fernanda Santos; ROSSI-BARBOSA, Luiza Augusta Rosa. A importância da capacitação em saúde auditiva: uma revisão integrativa. Revista CEFAC, São Paulo, v. 16, n. 4, p.1318-1325, Ago. 2014.

ROSA, Jonathan; BARTH, Priscila Orlandi.; GERMANI, Alessandra Regina Muller A sala de espera no agir em saúde: espaço de educação e promoção à saúde. Perspectiva. Erechim, v. 35, n.129, p. 121-130, mar. 2011.

ROSSI-BARBOSA, Luiza Augusta Rosa et al. Processo de construção de indicadores em saúde auditiva. Revista CEFAC, São Paulo, v. 13, n. 1, p. 102-111, Fev. 2011.

SANTOS, Juliana Nunes et al. Percepção de agentes comunitários de saúde sobre os riscos à saúde fonoaudiológica. Revista da Sociedade Brasileira de Fonoaudiologia, São Paulo, v. 17, n. 3, p. 333-339, 2012.

SILVA, Talita Naiara Rossi da et al. Sala de espera: uma possibilidade de intervenção em Saúde do Trabalhador. Cadernos Brasileiros de Terapia Ocupacional, São Carlos, v. 27, n.4, p. 907-916, Dez. 2019.

SCALABRIN, Izabel Cristina; MOLINARI, Adriana Maria Corder. A importância da prática do estágio supervisionado nas licenciaturas. Revista Unar, v.7, n.1, p. 1-12, 2013.

TEIXEIRA, Enéas Rangel; VELOSO, Raquel Coutinho. O grupo em sala de espera: território de práticas e representações em saúde. Texto contexto - enfermagem, Florianópolis, v. 15, n. 2 , p. 320-325, Jun 2006.

ZAMBENEDETTI, Gustavo. Sala de espera como estratégia de educação em saúde no campo da atenção às doenças sexualmente transmissíveis. Saúde e sociedade, São Paulo, v. 21, n. 4, p. 1075-1086, Dez. 2012. 